\title{
Interleukin-12 exacerbates symptoms in an MRL/MpJ-Faslpr mouse model of systemic lupus erythematosus
}

\author{
LING LI $^{1 *}$, XIAOJUN SUN ${ }^{1 *}$, SISI WU ${ }^{2}$, XIN YUAN ${ }^{1}$, BINGXIN LIU $^{3}$ and XUEPING ZHOU ${ }^{4}$ \\ ${ }^{1}$ Department of Rheumatology, Taizhou Hospital Affiliated to Nanjing University of Chinese Medicine, \\ Taizhou, Jiangsu 225300; ${ }^{2}$ Medical Intensive Care Unit, Ningbo Women and Children's Hospital, Ningbo, Zhejiang 315000; \\ ${ }^{3}$ Department of Rheumatology, Jiangsu Taizhou People's Hospital, Taizhou, Jiangsu 225300; \\ ${ }^{4}$ Institute of Acute Disorders of Traditional Chinese Internal Medicine, The First Clinical College of Nanjing \\ University of Chinese Medicine, Nanjing University of Chinese Medicine, Nanjing, Jiangsu 210023, P.R. China
}

Received August 11, 2020; Accepted January 28, 2021

DOI: $10.3892 /$ etm.2021.10059

\begin{abstract}
Interleukin (IL)-12 modulates the generation and function of a variety of immune cells and serves an important role in the pathogenesis of autoimmune diseases. However, the precise role of IL-12 in the pathogenesis of systemic lupus erythematosus (SLE) remains to be elucidated. In the present study, the serum levels of IL-12 in patients with SLE were determined using an ELISA. The association between serum levels of IL-12 and clinical and laboratory indices, specifically, disease activity and complement 3 , were analyzed. Recombinant IL-12 or an anti-IL-12 antibody was used to treat the MRL/ MpJ-Fas ${ }^{\mathrm{lpr}}$ mouse model of systemic lupus erythematosus. The glomerulonephritis and inflammatory cell infiltration was examined to evaluate histological changes using hematoxylin and eosin and Periodic acid-Schiff staining. Serum creatinine and proteinuria were used to determine renal function. The levels of anti-double stranded DNA and anti-nuclear autoantibodies were assessed. The results demonstrated that serum levels of IL-12 were markedly increased in patients with SLE compared with controls and in lupus model mice in comparison with control mice. The serum levels of IL-12 increased with disease severity in patients with SLE. SLE-like symptoms were exacerbated in lupus model mice treated with exogenous IL-12. However, SLE-like symptoms were ameliorated in lupus model mice treated with an anti-IL-12 antibody. The present results demonstrated that IL-12 aggravated SLE and anti-IL12
\end{abstract}

Correspondence to: Professor Xueping Zhou, Institute of Acute Disorders of Traditional Chinese Internal Medicine, The First Clinical College of Nanjing University of Chinese Medicine, Nanjing University of Chinese Medicine, 138 Xianlin Dadao Road, Nanjing, Jiangsu 210023, P. R. China

E-mail: zhouxueping789@163.com

*Contributed equally

Key words: interleukin 12, systemic lupus erythematosus, cytokines, lupus nephritis, renal injury antibodies ameliorated SLE. The present data suggest that blocking IL-12 may be a beneficial therapeutic strategy to halt the progression of lupus nephritis.

\section{Introduction}

Systemic lupus erythematosus (SLE) is a systemic autoimmune disease that may injure the kidneys, joints, blood vessels, lungs, heart and skin (1). Lupus nephritis (LN), inflammation of the kidney due to lupus, is one of the most severe complications seen in patients with SLE. Furthermore, 50-60\% of patients with SLE exhibit renal symptoms within 10 years of the onset of disease (2). LN is characterized by progressive renal function decline, microscopic hematuria and proteinuria (3).

The pathogenesis of $\mathrm{LN}$ is associated with multiple factors, including sex, steroids and excess salt intake, which lead to the malfunction of immune self-tolerance and the development of autoimmunity $(4,5)$. Evidence from both human and experimental models supports the hypothesis that cytokine dysregulation contributes to kidney diseases, including LN (6,7). Among these cytokines, IL-12 is an important mediator of immunity that has been implicated in the pathogenesis of SLE. IL-12 is secreted by innate immune cells upon microbial stimulation $(8,9)$. Studies have demonstrated that IL-12 is a pro-inflammatory cytokine that can promote $\mathrm{T}$ helper 1 (Th1) and $\mathrm{T}$ follicular helper cell differentiation. Studies have also reported that IL-12 is essential for cytotoxic $\mathrm{T}$ cell activation and function $(8,10)$. Additionally, studies have demonstrated that IL-12 could be an effective therapeutic target for psoriasis, Crohn's disease and rheumatoid arthritis $(11,12)$. Vom Berg et al (13) found that inhibition of IL-12/IL-23 signaling reduced Alzheimer's disease-like pathology and cognitive decline. An increase in IL-12 concentration has been observed in patients with SLE compared with healthy controls, and this is positively associated with the SLE disease activity index (SLEDAI), a clinical assessment of lupus disease activity in the preceding 10 days (14). However, Huang et al (15) suggested that IL-12 expression is reduced in patients with SLE in comparison with controls. Therefore, the precise role of IL-12 in the pathogenesis of SLE remains to be elucidated. 
To understand the role of IL-12 in the pathogenesis of LN, the serum levels of IL-12 in patients with SLE and mice were determined. The lupus model mice were treated with recombinant IL-12 or an anti-IL-12 antibody. It was revealed that serum levels of IL-12 were markedly increased in patients with SLE. Exogenous IL-12 exacerbated LN-like symptoms in lupus model mice.

\section{Materials and methods}

Patients and healthy controls. A total of 30 patients with SLE and 30 healthy volunteers were recruited at Taizhou Hospital Affiliated to Nanjing University of Chinese Medicine (Taizhou, China) between January 2018 and December 2019 for the present study. All patients were diagnosed with SLE according to the criteria set out by the American College of Rheumatology, which were revised in 1997, and all underwent a renal biopsy prior to the study, in order to reveal cases of LN according to the International Society of Nephrology/ Renal Pathology Society 2003 classification (16,17). The inclusion criteria were as follows: i) Patients aged 18 years; and ii) patients with complete documented data. The exclusion criteria were as follows: i) Infections, malignancies and other inflammatory diseases; and ii) lack of key information that rendered evaluation impossible. The demographic and clinical characteristics of the patients with SLE are shown in Table I. The patients with SLE were divided into two groups according to their SLEDAI score (17): Inactive patients, $<5$; active patients, $\geq 5$. Complement 3 and 4 (C3 and C4) was determined by biochemical analyzer (Hitachi, Ltd.). The anti-anti-nuclear antibody (ANA) antibody ELISA kit was from Zeus Scientific Inc. (cat. no. 2Z29001G). Patient serum was prepared with 1:21 dilution, following which $100 \mu 1$ sample was added to each well coated with inactivated antigen and incubated for $60 \mathrm{~min}$ at $20-25^{\circ} \mathrm{C}$. After washing, the horseradish peroxidase (HRP)-conjugated goat anti-human IgG was added for $30 \mathrm{~min}$ at $20-25^{\circ} \mathrm{C}$. After washing, 3,3',5,5'-Tetramethylbenzidine substrate solution (TMB) was added for $30 \mathrm{~min}$ at $20-25^{\circ} \mathrm{C}$. Finally, the stop solution $\left(1 \mathrm{M} \mathrm{H}_{2} \mathrm{SO}_{4}, 0.7 \mathrm{MHCl}\right)$ was added and absorbance was measured using a microplate reader at a wavelength of $450 \mathrm{~nm}$.

The anti-double-stranded DNA (ds-DNA) ELISA kit was from Zeus Scientific Inc. (cat. no. 2Z2881G). The serum samples from each patient was prepared in a 1:21 dilution before $100 \mu \mathrm{l}$ diluted serum was added each well coated with inactivated antigen and incubated for $30 \mathrm{~min}$ at $20-25^{\circ} \mathrm{C}$. After washing, the HRP-conjugated goat anti-human IgG was added for $30 \mathrm{~min}$ at $20-25^{\circ} \mathrm{C}$. After washing, TMB was added for $30 \mathrm{~min}$ at $20-25^{\circ} \mathrm{C}$. Finally, the stop solution $(1 \mathrm{M}$ $\mathrm{H}_{2} \mathrm{SO}_{4}, 0.7 \mathrm{MHCl}$ ) was added. A microplate reader was used to read absorbance in each well at a wavelength of $450 \mathrm{~nm}$. The calibrator within this ELISA kit has been assigned with both a correction factor for the generation of index values and a calibrator value for the generation of unit values. According to the interpretation of results of the anti-ANA and anti-dsDNA ELISA kits, the serum in each patient was defined as negative (anti-ANA', anti-ds-DNA') or positive (anti-ANA ${ }^{+}$, anti-ds-DNA ${ }^{+}$. The anti-ANA ${ }^{+}(\%)$ in Table I was referring to patients with anti-ANA ${ }^{+}$among all patients. The Anti-dsDNA ${ }^{+}$ (\%) in Table I was referring to patients with Anti-dsDNA ${ }^{+}$ among all patients. Written informed consent was obtained from each participant. The present study was approved by the Ethics Committee of Taizhou Hospital Affiliated to Nanjing University of Chinese Medicine (Taizhou, China).

Mice. In total, 36 female MRL/MpJ-Fas ${ }^{\mathrm{lpr}}$ and six female C57BL/6 mice were obtained from the Laboratory Animal Center, Academy of Military Medical Sciences (Beijing, China). MRL/MpJ-Fas ${ }^{\text {lpr }}$ mice develop an autoimmune disease resembling SLE, including an increase in anti-double-stranded DNA (anti-dsDNA) antibodies in the blood, and develop severe nephritis (18). All the mice used in this study were housed at room temperature $\left(20-24^{\circ} \mathrm{C}\right)$ and $45-60 \%$ humidity in a $12-\mathrm{h}$ light/dark cycle. Mice had access to diet and water ad libitum. Animal experiments were performed in accordance with the guidelines and approved by the Committee of Experimental Animal Administration of Taizhou Hospital Affiliated to Nanjing University of Chinese Medicine (Taizhou, China).

Recombinant IL-12 or anti-IL-12 antibody treatment of MRL/ MpJ-Fas ${ }^{l p r}$ mice. Six female 16-week-old MRL/MpJ-Fas ${ }^{1 p r}$ mice (35-40 g) were injected intraperitoneally with murine recombinant IL-12 (100 $\mu \mathrm{g} / \mathrm{kg}$ body weight; cat. no. 419-ML050/CF; R\&D Systems, Inc.) for 7 consecutive days. Six 16-week-old MRL/MpJ-Fas ${ }^{\mathrm{lpr}}$ mice of the same age (35-40 g) that received an equal volume of PBS served as controls. For anti-IL-12 treatment, six female 16-week-old MRL/ $\mathrm{MpJ} J-F a s^{\mathrm{lpr}}$ mice were injected intraperitoneally with the antiIL-12 antibody (5 $\mu \mathrm{g} / \mathrm{g}$ body weight, cat. no. MAB419-100; R\&D Systems, Inc.) once. Six female mice of the same age received an equal volume of rat $\mathrm{IgG} 2 \mathrm{a}$ isotype antibody (cat. no. MAB005R, R\&D Systems, Inc.) served as controls.

ELISA for IL-12. Whole blood was collected from patients with SLE, healthy controls and mice. The blood was immediately centrifuged at $500 \mathrm{x}$ g for $5 \mathrm{~min}$ at $4^{\circ} \mathrm{C}$ for isolation of serum. Subsequently, the serum was stored at $-80^{\circ} \mathrm{C}$. All serum samples were brought to room temperature before the assay. Human (cat. no. D1200) and mouse (cat. no. M1270) IL-12 ELISA kits were purchased from R\&D Systems, Inc. All experiments were performed according to the manufacturer's protocols.

Anti-dsDNA antibody. Anti-dsDNA antibodies in serum from mice were detected as previously described (19). Briefly, 96-well microtiter plates were coated with $50 \mu \mathrm{g} / \mathrm{ml}$ calf thymus dsDNA (Sigma-Aldrich; Merck KGaA) overnight at $4^{\circ} \mathrm{C}$. After blocking with $1 \%$ BSA (Sigma-Aldrich; Merck KGaA) in PBS at room temperature for $1 \mathrm{~h}, 100$-fold diluted serum $(100 \mu \mathrm{l})$ was added, followed by incubation at room temperature for $2 \mathrm{~h}$. Subsequently, goat anti-mouse antibody conjugated to horseradish peroxidase (1:2,000; cat. no. SA00001-1; Proteintech Group, Inc.) was added, followed by incubation at room temperature for $1 \mathrm{~h}$. Next, 3,3',5,5'-Tetramethylbenzidine substrate solution (cat. no. B019-1-1; Nanjing Jiancheng Bioengineering Institute)was added, and plates were incubated at room temperature for $30 \mathrm{~min}$. Finally, stop solution ( $1 \mathrm{M} \mathrm{H}_{2} \mathrm{SO}_{4}$ ) was added and the absorbance in each well was monitored at $450 \mathrm{nmin}$ a microplate reader (ELX808; BioTek China). 
Table I. Characteristics of patients with SLE.

\begin{tabular}{|c|c|c|c|}
\hline Characteristic & Patients with SLE & Healthy control & P-value \\
\hline $\mathrm{N}$ & 30 & 30 & $\mathrm{~N} / \mathrm{A}$ \\
\hline Age, years & $29.13 \pm 7.24$ & $31.34 \pm 5.41$ & $>0.05$ \\
\hline Sex, male/female & $3 / 27$ & $5 / 25$ & $>0.05$ \\
\hline Disease duration, months, mean $\pm \mathrm{SD}$ (range) & $37.04 \pm 16.04(1-234)$ & ND & N/A \\
\hline SLEDAI & $8.71 \pm 3.12$ & ND & $\mathrm{N} / \mathrm{A}$ \\
\hline Scr $(\mathrm{mg} / \mathrm{dl})$ & $1.69 \pm 0.22$ & $1.36 \pm 0.19$ & $<0.05$ \\
\hline Proteinuria (g/day) & $3.44 \pm 0.53$ & ND & N/A \\
\hline Serum albumin $(\mathrm{g} / \mathrm{l})$ & $29.84 \pm 1.52$ & $43.34 \pm 2.11$ & $<0.01$ \\
\hline Anti-dsDNA $^{+}(\%)$ & 76.52 & ND & N/A \\
\hline Anti-ANA $^{+}(\%)$ & 86.44 & ND & N/A \\
\hline $\mathrm{C} 3(\mathrm{~g} / \mathrm{l})$ & $0.57 \pm 0.17$ & $0.91 \pm 0.22$ & $<0.01$ \\
\hline $\mathrm{C} 4(\mathrm{~g} / \mathrm{l})$ & $0.17 \pm 0.06$ & $0.49 \pm 0.12$ & $<0.01$ \\
\hline Mean blood pressure (mmHg) & $97.05 \pm 11.04$ & $86.06 \pm 16.08$ & $>0.05$ \\
\hline $\mathrm{Hb}(\mathrm{g} / \mathrm{dl})$ & $8.91 \pm 2.32$ & $15.33 \pm 1.74$ & $<0.01$ \\
\hline Urine $\mathrm{RBC}$ count $\left(10^{4} / \mathrm{ml}\right)$ mean $\pm \mathrm{SD}$ (range) & $349.03 \pm 105.02(2-2300)$ & ND & $\mathrm{N} / \mathrm{A}$ \\
\hline
\end{tabular}

SLE, systemic lupus erythematosus; SLEDAI, systemic lupus erythematosus disease activity index; Scr, serum creatinine; Anti-dsDNA, antidouble-stranded DNA antibody; ANA, antinuclear antibody; Hb: hemoglobin; RBC, red blood cell; ND, not determined; N/A, not applicable.

Serum creatinine level and proteinuria analysis. The creatinine levels in the serum of mice were measured using quantitative enzyme colorimetric kits (cat. no. C011-2-1; Nanjing Jiancheng Bioengineering Institute) according to the manufacturer's protocols. The random urine of the mice was collected and urine protein was determined using a Bradford Protein Assay Kit (Nanjing KeyGen Biotech Co., Ltd.). Urinary albumin was determined using an Albumin Assay kit (cat. no. E038-1-1; Nanjing Jiancheng Bioengineering Institute).

Histopathological analysis of kidney samples. The mice were anesthetized with sodium pentobarbital (200 mg/kg i.p.) before being sacrificed. The kidneys of the mice were collected and fixed in $4 \%$ paraformaldehyde at room temperature for $24 \mathrm{~h}$. Subsequently, the kidneys were embedded in paraffin, cut into 3- $\mu \mathrm{m}$-thick sections, dehydrated in an ascending ethanol gradient and xylene and stained with hematoxylin and eosin (H\&E, at room temperature for $5 \mathrm{~min}$ ) according to standard procedures.

For periodic acid-Schiff (PAS) staining, the PAS staining kit was used according to manufacturer's protocol (cat. no. D004-1-2; Nanjing Jiancheng Bioengineering Institute). The sections were first dewaxed with xylene and rehydrated in a descending ethanol gradient. The sections were then oxidized with $10 \mathrm{~g} / \mathrm{l}$ period ate for $15 \mathrm{~min}$ at $20-25^{\circ} \mathrm{C}$. Next, the sections were stained with Schiff's reagent for $45 \mathrm{~min}$ at $20-25^{\circ} \mathrm{C}$. The $20 \mathrm{~g} / \mathrm{l}$ methyl green was used for counterstaining for $15 \mathrm{~min}$ at $20-25^{\circ} \mathrm{C}$. The histopathological analysis of the kidney samples was performed by two pathologists in a blinded manner with an optical light microscope (10 fields for each sample, magnification, x20, Olympus BH-2; Olympus Corporation).

RNA extraction and reverse transcription-quantitative PCR of $I L-12$. Total RNA was extracted from peripheral blood mononuclear cells and kidney tissues of mice using an RNA isolation kit (cat. no. 9108Q; Takara Bio, Inc.). The peripheral blood mononuclear cells were isolated from mice by FicollPaque density gradient centrifugation (cat. no. LTS1092, Tianjin Haoyang Biological Manufacture, Co., Ltd.) before the samples were centrifuged at $400 \mathrm{x}$ g for $20 \mathrm{~min}$ in $25^{\circ} \mathrm{C}$. The kidney homogenate was grounded using glass homogenizer. Complementary DNA was synthesized using PrimeScript ${ }^{\mathrm{TM}}$ RTMaster Mix kit (cat. no. RR036Q; Takara Bio, Inc.) at $37^{\circ} \mathrm{C}$ for $15 \mathrm{~min}$ and $85^{\circ} \mathrm{C}$ for $5 \mathrm{sec}$. The IL-12 mRNA was detected using TB Green Premix Ex Taq (Tli RNase H Plus) kit (Takara Bio, Inc.) in an Applied Biosystems 7500 PCR system (Applied Biosystems; Thermo Fisher Scientific, Inc.). The RT-qPCR cycling program was set at one cycle of pre-denaturation at $95^{\circ} \mathrm{C}$ for $5 \mathrm{~min}$, followed by 40 cycles at $95^{\circ} \mathrm{C}$ for $10 \mathrm{sec}$ and $60^{\circ} \mathrm{C}$ for $1 \mathrm{~min}$. The primers were synthesized by Takara Bio, Inc. The relative expression of IL-12 was determined and normalized to the expression of housekeeping gene GAPDH and calculated with the $2^{-\triangle \Delta C q}$ assay (20). The primers of mouse IL-12 were as follows Forward, 5'-ACCCTGACCATCACTGTCAA-3' and reverse, 5'-GTGGAGCAGCAGATGTGAGT-3' and GAPDH forward, 5'-ACAACTTTGGCATTGTGGAA-3' and reverse, 5'-GATGCAGGGATGATGTTCTG-3'.

Statistical analysis. Data are presented as the mean \pm SEM. For the comparison of means between two groups, two-tailed unpaired t-tests were performed. Categorical variables are presented as frequencies and percentages. Fisher's exact test was used to compare categorical variables, such as the sex distribution. Correlations between values were analyzed using Spearman's test. All statistical calculations were performed using GraphPad Prism software (version 7.0; GraphPad Software, Inc.). $\mathrm{P}<0.05$ was considered to indicate a statistically significant difference. 
A

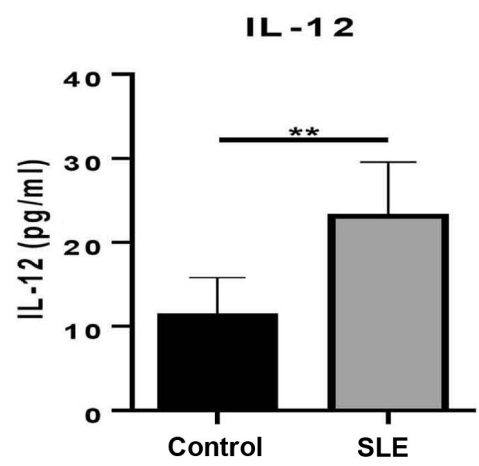

C

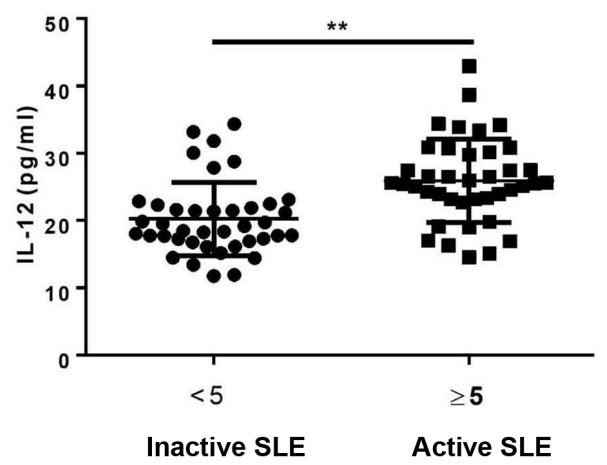

B

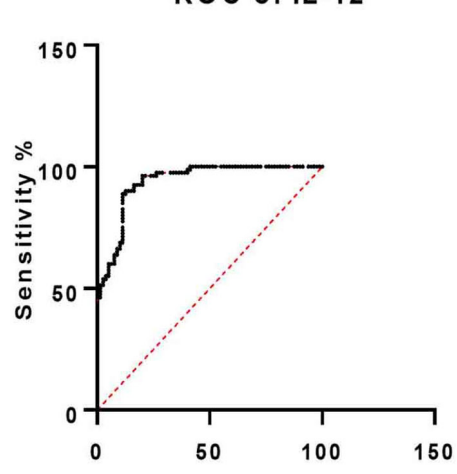

D $100 \%$ - specificity \%

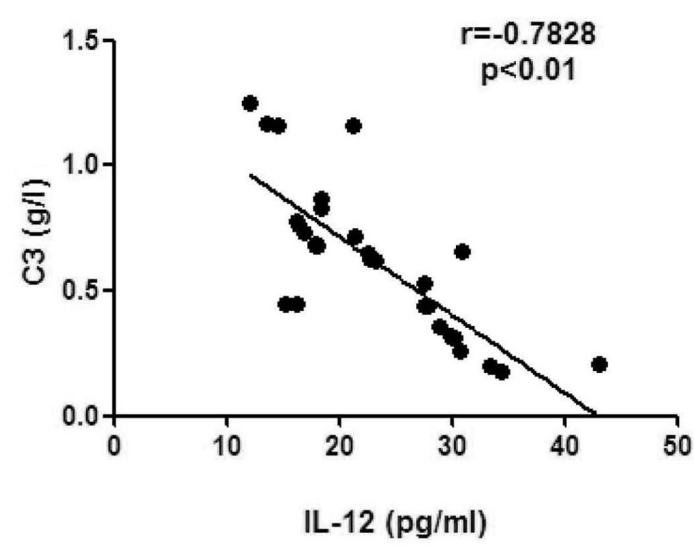

Figure 1. Serum levels of IL-12 are increased and are positively associated with disease activity in patients with SLE. (A) Serum levels of IL-12 in patients with SLE. (B) Receiver operating characteristic curve for IL-12 in the evaluation of patients with SLE, and the optimal cut-off value based on the Youden index. (C) Serum levels of IL-12 were evaluated in patients with SLE according to disease activity. (D) Association between IL-12 and C3 in the serum of patients with SLE. ${ }^{* *} \mathrm{P}<0.01$. IL, interleukin; SLE, systemic lupus erythematosus.

\section{Results}

Characteristics of patients with SLE. A total of 30 patients diagnosed with SLE were recruited in the present study. Table I summarizes the sex, age, SLE disease history and clinical symptoms of the patients. The mean age was $29.1 \pm 7.2$ years (range, 21-57 years). The median SLE disease duration since diagnosis was 37 months (range, 1-234 months). The SLEDAI score was 7 (range, 4-23). Among the patients, $76.5 \%$ were anti-dsDNA-positive and $86.4 \%$ were anti-antinuclear antibody-positive (Table I). All patients with SLE received standard therapy, including steroids and immunosuppressive after presentation in the hospital.

Serum levels of IL-12 are elevated in patients with SLE. To determine whether IL-12 was involved in the pathogenesis of SLE, the IL-12 levels in serum from patients with SLE and healthy controls were measured. The patients with SLE had significantly increased serum levels of IL-12 compared with healthy controls $(23.09 \pm 6.47$ vs. $11.26 \pm 4.58 \mathrm{pg} / \mathrm{ml}$; Fig. $1 \mathrm{~A})$. By constructing an empirical receiver operating characteristic (ROC) curve, the optimal cut-off value of $22.41 \mathrm{pg} / \mathrm{ml}$ was selected according to the Youden index, which gave a sensitivity and specificity of 51.25 and $98.75 \%$, respectively (Fig. 1B). The area under the ROC curve was 0.9392 . To determine the clinical significance of IL-12, patients with SLE were divided into active and inactive groups according to their SLEDAI score. The correlation between serum IL-12 and SLEDAI was assessed, however no significant correlation was identified (data not shown). It was established that the levels of IL-12 in patients with active SLE $(25.93 \pm 6.21 \mathrm{pg} / \mathrm{ml})$ were higher than those in patients with inactive SLE $(20.25 \pm 5.45 \mathrm{pg} / \mathrm{ml}$; Fig. 1C). As hypocomplementemia is one of the immunological abnormalities in patients with SLE (3), the associations among IL-12, C3 and C4 were evaluated. The results revealed that serum levels of IL-12 were negatively associated with C3 in patients with SLE (Fig. 1D), whilst serum levels of IL-12 exhibited no significant association with $\mathrm{C} 4$ (data not shown). The relationship between IL-12 and titers of anti-dsDNA antibody was also analyzed. However, no significant correlation was found (data not shown). These findings indicated that serum levels of IL-12 may reflect the severity of symptoms in patients with SLE.

IL-12 levels are increased in a mouse model of lupus. The levels of IL-12 in the lupus-model MRL/MpJ-Fas ${ }^{\mathrm{lpr}}$ mice were examined and compared with those in control mice. It was identified that the serum levels of IL-12 were markedly increased in the MRL/MpJ-Fas ${ }^{\mathrm{lpr}}$ mice $(21.30 \pm 2.73 \mathrm{pg} / \mathrm{ml})$ compared with control C57/BL6 mice $(8.11 \pm 0.72 \mathrm{pg} / \mathrm{ml}$; Fig. 2A). IL-12 mRNA expression in the peripheral blood mononuclear cells of MRL/MpJ-Fas ${ }^{1 p r}$ mice was also increased 
A

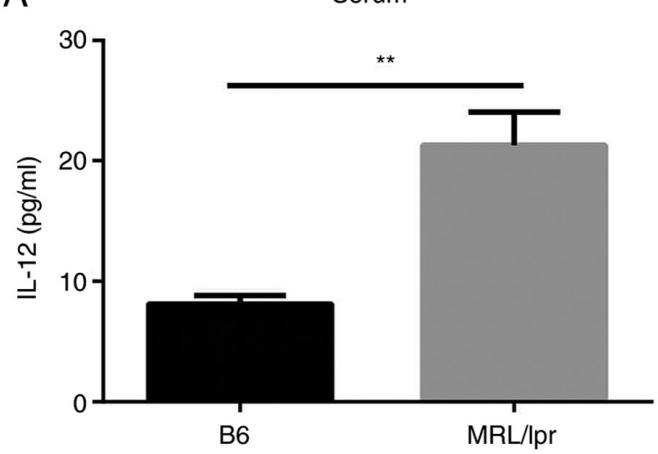

C

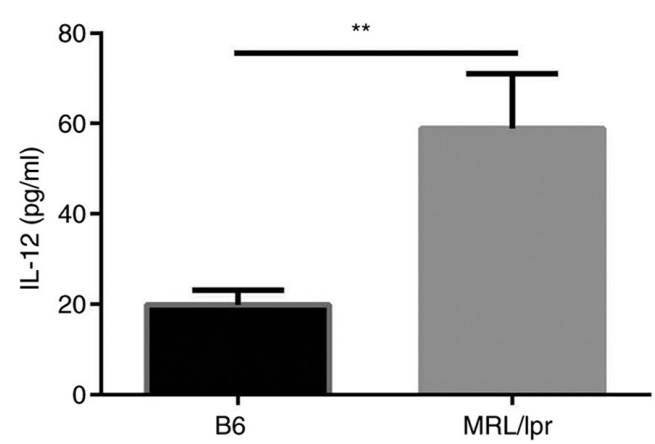

B

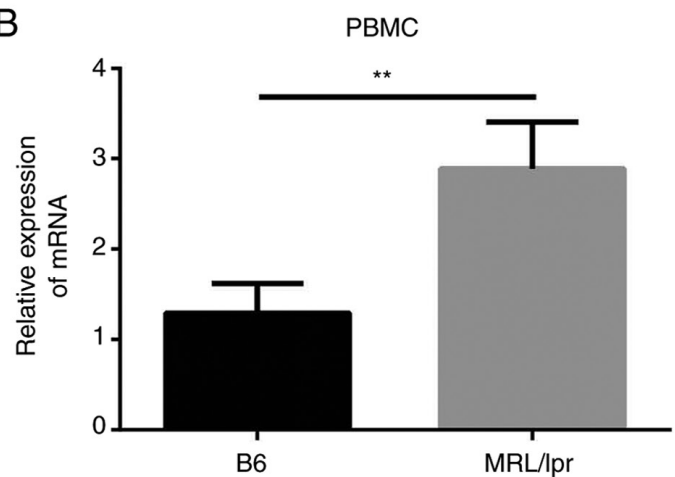

D

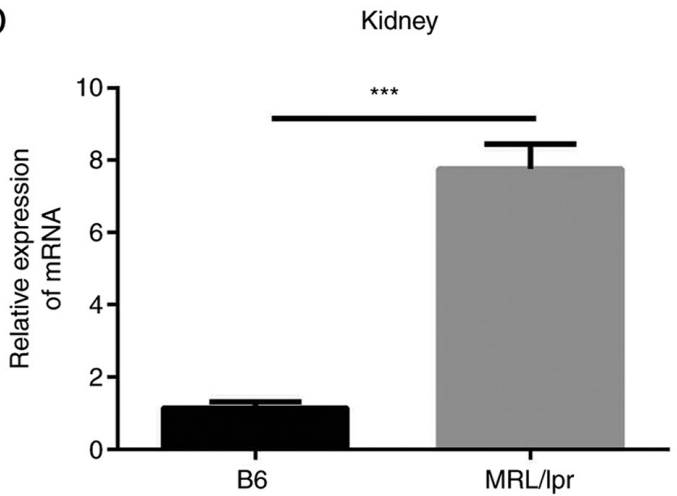

Figure 2. IL-12 expression is increased in lupus-model MRL/MpJ-Fas ${ }^{\mathrm{lpr}}$ mice. (A) Serum levels of IL-12 in MRL/MpJ-Fas ${ }^{\mathrm{lpr}}$ mice in comparison with C57BL/6 mice. (B) IL-12 mRNA expression in PBMCs of MRL/MpJ-Fas ${ }^{\text {lpr }}$ mice in comparison with C57BL/6 mice. (C) IL-12 expression in renal homogenate from MRL/MpJ-Fas ${ }^{\mathrm{lpr}}$ mice in comparison with C57BL/6 mice. (D) IL-12 mRNA expression in kidney samples from MRL/MpJ-Fas ${ }^{\mathrm{lpr}}$ mice in comparison with C57BL/6 mice. ${ }^{* *} \mathrm{P}<0.01$ and ${ }^{* * *} \mathrm{P}<0.001$. IL, interleukin; PBMCs, peripheral blood mononuclear cells.

compared with that in control mice (Fig. 2B). Subsequently, the mRNA and protein expression levels of IL-12 in the kidneys of mice were examined. The results revealed that IL-12 levels in renal homogenate from MRL/MpJ-Fas ${ }^{\mathrm{lpr}}$ mice $(58.94 \pm 6.99 \mathrm{pg} / \mathrm{ml})$ were increased compared with those in control mice (19.82 $\pm 1.90 \mathrm{pg} / \mathrm{ml}$; Fig. 2C). Additionally, IL-12 mRNA expression in kidneys tissue from MRL/MpJ-Fas ${ }^{\mathrm{lpr}}$ mice was increased (Fig. 2D). These findings suggested that IL-12 levels were increased in lupus-model MRL/MpJ-Fas ${ }^{\mathrm{lpr}}$ mice in comparison with controls.

Exogenous IL-12 aggravates LN. MRL/MpJ-Fas ${ }^{\mathrm{lpr}}$ mice (age, 16 weeks) were injected with recombinant mouse IL-12. It was revealed that the pathology of the kidney deteriorated following this injection, as indicated by increased mesangial cell proliferation and mesangial matrix deposition in glomeruli (Fig. 3A), and interstitial cellular infiltration (Fig. 3B). Levels of the anti-dsDNA antibody, one of the hallmarks of SLE (2), were demonstrated to be increased following exogenous IL-12 treatment in comparison with PBS treatment (Fig. 3C). Compared with control treated mice, the MRL/MpJ-Fas ${ }^{\text {lpr }}$ mice treated with recombinant murine IL-12 exhibited higher levels of serum creatinine $(4.45 \pm 0.37 \mathrm{vs} .6 .31 \pm 0.38 \mu \mathrm{mol} / \mathrm{l})$ and proteinuria $(5.95 \pm 0.16$ vs. $7.32 \pm 0.21 \mathrm{mg} / \mathrm{ml}$; Fig. 3D and E). These results highlighted that exogenous IL-12 treatment exacerbated the SLE-like symptoms in MRL/MpJ-Fas ${ }^{\mathrm{lpr}}$ mice.

Anti-IL-12 antibody treatment improves $L N$. To verify the effects of IL-12 on the pathogenesis of SLE, MRL/MpJ-Fas ${ }^{\text {Ipr }}$ mice were treated with an anti-IL-12 antibody. The histological results demonstrated that anti-IL-12 treatment alleviated the kidney injury, such as mesangial cell proliferation and mesangial matrix deposition in glomeruli (Fig. 4A), and interstitial cellular infiltration (Fig. 4B). Additionally, the serum levels of anti-dsDNA antibody were identified to be decreased in the anti-IL-12 treatment group in comparison with the mice treated with an isotype control (Fig. 4C). Compared with that in mice in the isotype control group, the concentration of serum creatinine was decreased in mice in the anti-IL-12 treatment group $(4.23 \pm 0.35$ vs. $2.99 \pm 0.27 \mu \mathrm{mol} / 1$; Fig. 4D). In addition, the levels of proteinuria were reduced in mice in the anti-IL-12 treatment group in comparison with the isotype control $(7.20 \pm 0.20$ vs. $5.19 \pm 0.15 \mathrm{mg} / \mathrm{ml}$; Fig. 4E). These findings indicated that anti-IL-12 treatment ameliorated LN-like symptoms in MRL/MpJ-Fas ${ }^{\mathrm{lpr}}$ mice.

\section{Discussion}

The precise role of IL-12 in the pathogenesis of LN is not fully understood $(8,10)$. The present study revealed that serum levels of IL-12 were increased in patients with lupus and in lupus-model mice in comparison with controls. IL-12 reflected the disease severity of patients with SLE using the SLEDAI score. The present results suggested that IL-12 may serve an important role in SLE. Several lines of experimental evidence supported this conclusion. First, exogenous IL-12 treatment resulted in increased SLE-like symptoms in MRL/MpJ-Fas ${ }^{\mathrm{lpr}}$ mice in comparison with controls. Second, blocking of IL-12 
A

PBS

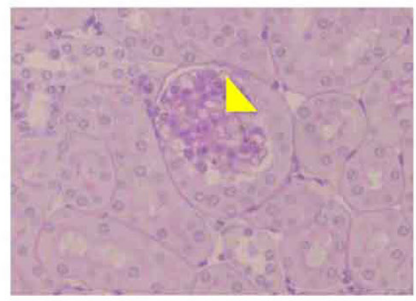

B

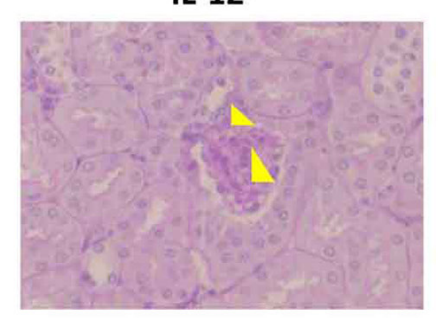

PBS

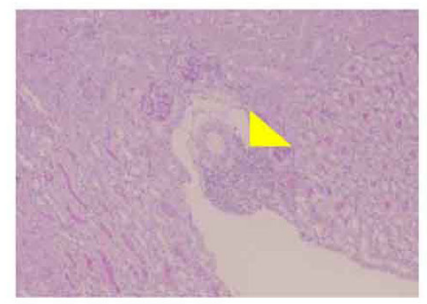

IL-12

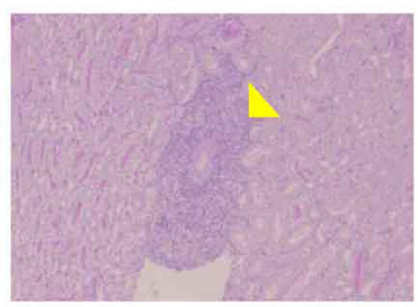

C

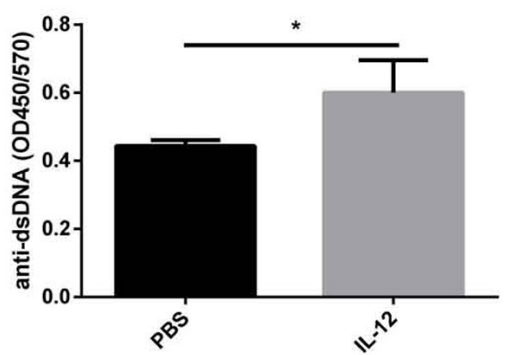

D

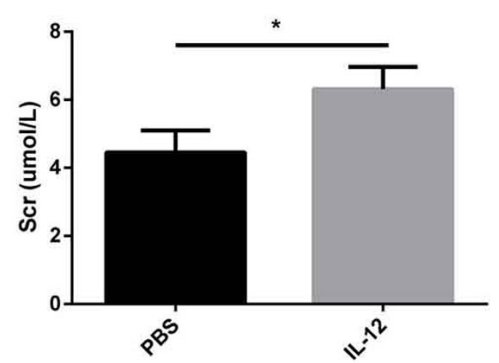

E

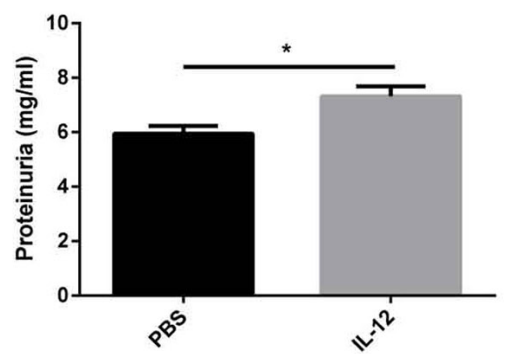

Figure 3. Exogenous IL-12 treatment aggravates lupus nephritis in MRL/MpJ-Fas ${ }^{\mathrm{lpr}}$ mice. (A) Representative images of H\&E staining of kidney samples from MRL/MpJ-Fas ${ }^{1 p r}$ mice treated with IL-12 or PBS. The mesangial cell proliferation and mesangial matrix deposition in glomeruli were indicated in yellow arrow. Magnification, x200.(B) Representative images of periodic acid-Schiff staining of kidney samples from MRL/MpJ-Fas ${ }^{\text {lpr }}$ mice treated with IL-12 or PBS. The interstitial cellular infiltration was indicated in yellow arrows. Magnification, x200. (C) Serum levels of anti-dsDNA antibody in mice treated with IL-12 or PBS. (D) Scr levels in mice treated with IL-12 or PBS. (E) Protein levels in urine of MRL/MpJ-Fas ${ }^{\mathrm{pr}}$ mice treated with IL-12 or PBS. IL, interleukin, anti-dsDNA, anti-double stranded DNA; scr, serum creatine. ${ }^{*} \mathrm{P}<0.05$.

A

Isotype antibody

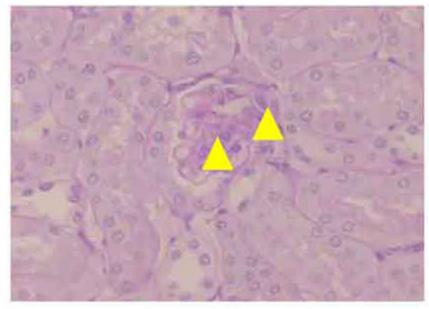

C

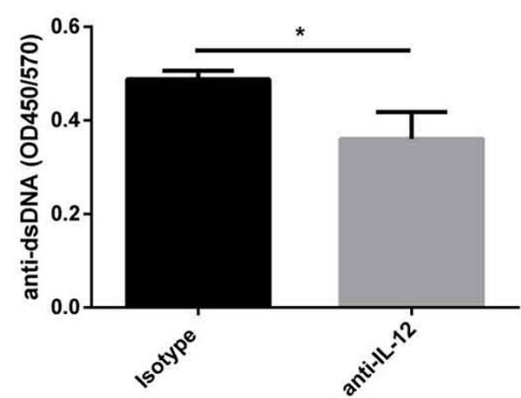

anti-IL-12

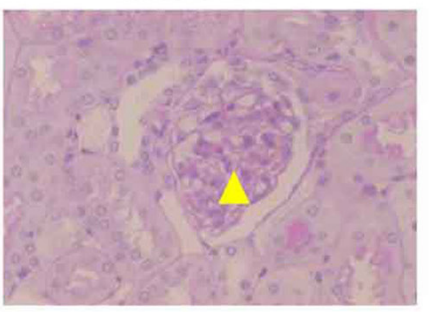

B

Isotype antibody

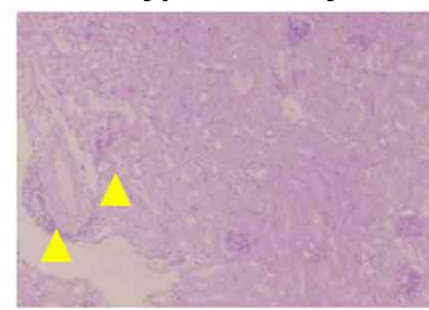

anti-IL-12

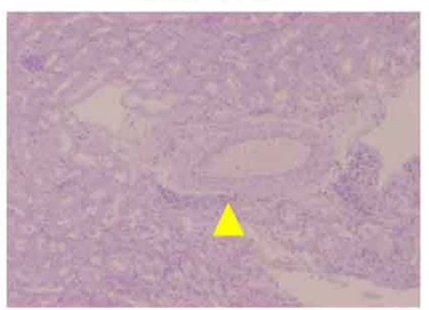

D

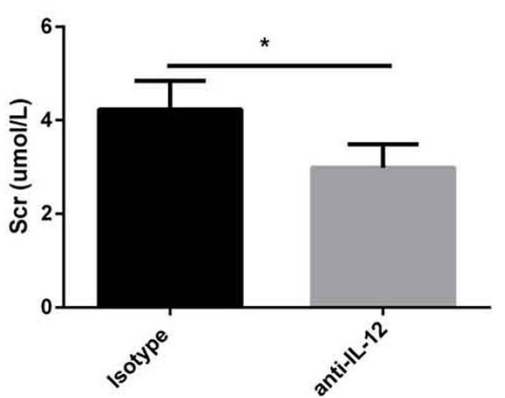

E

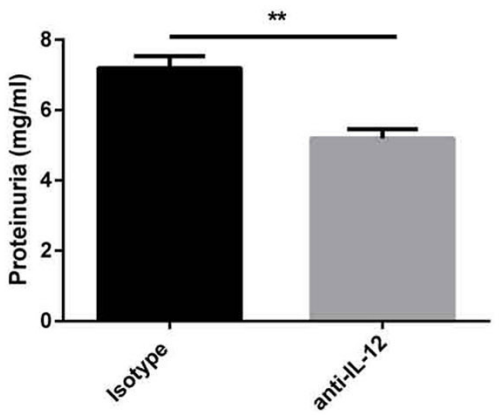

Figure 4. Anti-IL-12 antibody alleviates lupus nephritis in MRL/MpJ-Fas ${ }^{\mathrm{lpr}}$ mice. (A) Representative images of H\&E staining of kidney samples from MRL/ $\mathrm{MpJ}-F a s^{\mathrm{lpr}}$ mice treated with anti-IL-12 or PBS. The mesangial cell proliferation and mesangial matrix deposition in glomeruli were indicated in yellow arrow. Magnification, x200.(B) Representative images of periodic acid-Schiff staining of kidney samples from MRL/MpJ-Fas ${ }^{1 p r}$ mice treated with anti-IL-12 or PBS. The interstitial cellular infiltration was indicated in yellow arrows. Magnification, x200. (C) Serum levels of anti-dsDNA antibody in mice treated with anti-IL-12 or PBS. (D) Scr levels in mice treated with anti-IL-12 or PBS. (E) Protein levels in urine of MRL/MpJ-Faslprmice treated with anti-IL-12 or PBS. IL, interleukin, anti-dsDNA, anti-double stranded DNA; scr, serum creatine. ${ }^{*} \mathrm{P}<0.05$ and ${ }^{* * *} \mathrm{P}<0.01$.

with a monoclonal anti-IL-12 antibody improved SLE symptoms in MRL/MpJ-Fas ${ }^{\mathrm{lpr}}$ mice in comparison with controls.
SLE is a rheumatic disease characterized by loss of self-tolerance, leading to the development of autoantibodies 
against self-antigens. The autoantibodies and self-antigens form an immune complex and induce organ damage $(1,3)$. Previous studies have demonstrated that a variety of cytokines are involved in SLE $(2,21)$. IL-12 is a pro-inflammatory cytokine, largely produced by antigen-presenting cells (22). The pro-inflammatory capacity of IL-12 is ascribed to promotion of Th1 cell differentiation. Furthermore, IL-12 has been linked to innate immunity, as well as the development of adaptive immunity characterized by the induction of interferon- $\gamma$ production (9). IL-12 has been extensively studied in several animal models of autoimmune diseases and cancer. Trembleau et al (23) demonstrated that IL-12 accelerates the onset of autoimmune insulitis and diabetes. Studies have also demonstrated that exogenous administration of IL-12 causes severe manifestations of encephalomyelitis symptoms, while treatment with an anti-IL-12 antibody could ameliorate experimental autoimmune encephalomyelitis $(24,25)$. Previous studies have revealed that IL-12-deficient mice are protected from collagen-induced arthritis. The exogenous administration of IL-12 aggravates the disease, while inhibition of IL-12 by an anti-IL-12 antibody results in the reduced severity of collagen-induced arthritis $(26,27)$. However, the roles and mechanisms of IL-12 in SLE remain incompletely understood. Studies have demonstrated that IL-12 expression is elevated in the sera of patients with SLE commensurate with disease activity $(11,28)$. The present results are consistent with these findings of higher IL-12 levels in patients with SLE. However, a non-significant correlation existed between serum IL-12 levels and the SLEDAI. Patients were divided into two groups according to SLEDAI scores $(<5$, inactive patients; $\geq 5$, active patients). The IL-12 levels in patients with inactive SLE were lower than those in patients with active SLE, which suggested that IL-12 reflected the disease severity of patients with SLE. Furthermore, the present study revealed that the levels of IL-12 were negatively associated with C3 in serum, which was consistent with the findings of Lauwerys et al (29). However, Huang et al (15) reported that IL-12 specific subunit p35 mRNA expression in untreated and treated patients with SLE was lower than that in healthy controls and hypothesized that deficiency of IL-12 may contribute to the pathogenesis of SLE. Although the reasons for these different observations remain unknown, this may be because the mRNA expression was assessed in their study, while protein expression levels of IL-12 were determined in the present study.

The present study revealed that exogenous IL-12 treatment resulted in increased SLE-like symptoms in MRL/MpJ-Fas ${ }^{\mathrm{lpr}}$ mice in comparison with control treatment. In line with the present findings, Segal et al (30) found that administration of IL-12 to aging mice reversed the Th1/T helper 2 cytokine profile and, therefore, rendered them vulnerable to the induction of experimental SLE. However, injection of cDNA encoding IL-12 has been demonstrated to provoke limited amelioration in the pathogenesis of SLE model mice (31). The differences between the study by Neumann et al (31) and the present study may be ascribed to anti-IL-12 activity induced by the cDNA injection procedure, as the authors described.

In the present study, blocking of IL-12 with a monoclonal anti-IL-12 antibody improved SLE-like symptoms in MRL/MpJ-Fas ${ }^{\mathrm{lpr}}$ mice. A previous study demonstrated that MRL/MpJ-Fas ${ }^{\mathrm{lpr}}$ mice with a genetic deficiency in IL-12 have reduced kidney pathology, diminished lymphadenopathy and prolonged survival (32). Therefore, these findings indicated that eliminating IL-12 may reduce systemic pathology in lupus-model MRL/MpJ-Fas ${ }^{\mathrm{lpr}}$ mice. Increasing knowledge regarding IL-12 in SLE has led to the development of a novel therapeutic strategy targeting IL-12. Phase III clinical trials have demonstrated the efficacy of ustekinumab (a fully human IgG1 $\kappa$ mAb directed against the p40 subunit of IL-12 and IL-23) in treating moderate to severe plaque psoriasis (28). In a phase II randomized controlled trial in patients with active SLE, ustekinumab was superior to the placebo in terms of SLE Responder Index-4 response after 24 weeks (25). However, phase III clinical trials on the efficacy and safety of ustekinumab in patients with SLE are required.

In summary, the present study demonstrated that an increase in IL-12 reflected the disease severity in patients with SLE and mouse models. Exogenous administration of IL-12 aggravated manifestations of LN and treatment with neutralizing anti-IL-12 antibody ameliorated LN in lupusmodel MRL/MpJ-Fas ${ }^{\text {lpr }}$ mice in comparison with controls. The present data suggested that blocking IL-12 may be a therapeutic strategy to halt the progression of LN. However, there are certain unresolved questions concerning the mechanisms of IL-12 in LN that need to be addressed.

\section{Acknowledgements}

Not applicable.

\section{Funding}

The present study was supported by Taizhou Research and Development Program (grant no. TS201809).

\section{Availability of data and materials}

The datasets used and/or analyzed during the current study are available from the corresponding author on reasonable request.

\section{Authors' contributions}

LL and XS participated in study design, data collection, data analysis, data interpretation and drafting the paper. SW, XY and BL participated in patient recruitment, animal experiments and data collection. XZ supervised the whole research, designed the study, interpreted the data and wrote the paper. LL and XZ assessed the authenticity of all the raw data to ensure its legitimacy. All authors read and approved the final manuscript.

\section{Ethics approval and consent to participate}

This study was approved by Ethics Committee of Taizhou Hospital Affiliated to Nanjing University of Chinese Medicine (Taizhou, China). Written informed consents were obtained from all individuals.

\section{Patient consent for publication}

Not applicable. 


\section{Competing interests}

The authors declare that they have no competing interests.

\section{References}

1. Anders HJ, Saxena R, Zhao MH, Parodis I, Salmon JE and Mohan C: Lupus nephritis. Nat Rev Dis Primers 6: 7, 2020.

2. Stokes MB and D'Agati VD: Classification of lupus nephritis: Time for a change? Adv Chronic Kidney Dis 26: 323-329, 2019.

3. Davidson A, Aranow C and Mackay M: Lupus nephritis: Challenges and progress. Curr Opin Rheumatol 31: 682-688, 2019.

4. Song K, Liu L, Zhang X and Chen X: An update on genetic susceptibility in lupus nephritis. Clin Immunol 210: 108272, 2020.

5. Yang X, Yao G, Chen W, Tang X, Feng X and Sun L: Exacerbation of lupus nephritis by high sodium chloride related to activation of SGK1 pathway. Int Immunopharmacol 29: 568-573, 2015.

6. Frangou E, Georgakis S and Bertsias G: Update on the cellular and molecular aspects of lupus nephritis. Clin Immunol 216 $108445,2020$.

7. Tang Y, Zhang W, Zhu M, Zheng L, Xie L, Yao Z, Zhang H, Cao D and Lu B: Lupus nephritis pathology prediction with clinical indices. Sci Rep 8: 10231, 2018.

8. Trinchieri G: Interleukin-12 and the regulation of innate resistance and adaptive immunity. Nat Rev Immunol 3: 133-146, 2003.

9. Kang BY, Kim E and Kim TS: Regulatory mechanisms and their therapeutic implications of interleukin-12 production in immune cells. Cell Signal 17: 665-673, 2005.

10. Ma CS, Suryani S, Avery DT, Chan A, Nanan R, Santner-Nanan B Deenick EK and Tangye SG: Early commitment of naïve human CD4(+) $\mathrm{T}$ cells to the $\mathrm{T}$ follicular helper $(\mathrm{T}(\mathrm{FH})$ ) cell lineage is induced by IL-12. Immunol Cell Biol 87: 590-600, 2009.

11. Teng MWL, Bowman EP, McElwee JJ, Smyth MJ, Casanova JL, Cooper AM and Cua DJ: IL-12 and IL-23 cytokines: From discovery to targeted therapies for immune-mediated inflammatory diseases. Nat Med 21: 719-729, 2015.

12. Sandborn WJ, Gasink C, Gao LL, Blank MA, Johanns J, Guzzo C Sands BE, Hanauer SB, Targan S, Rutgeerts P, et al; CERTIFI Study Group: Ustekinumab induction and maintenance therapy in refractory Crohn's disease. N Engl J Med 367: 1519-1528, 2012.

13. Vom Berg J, Prokop S, Miller KR, Obst J, Kälin RE, LopateguiCabezas I, Wegner A, Mair F, Schipke CG, Peters O, et al: Inhibition of IL-12/IL-23 signaling reduces Alzheimer's diseaselike pathology and cognitive decline. Nat Med 18: 1812-1819, 2012.

14. Ueno H: The IL-12-STAT4 axis in the pathogenesis of human systemic lupus erythematosus. Eur J Immunol 50: 10-16, 2020.

15. Huang X, Hua J, Shen N and Chen S: Dysregulated expression of interleukin-23 and interleukin-12 subunits in systemic lupus erythematosus patients. Mod Rheumatol 17: 220-223, 2007.

16. Hochberg MC: Updating the American College of Rheumatology revised criteria for the classification of systemic lupus erythematosus. Arthritis Rheum 40: 1725-1726, 1997.

17. Weening JJ, D'Agati VD, Schwartz MM, Seshan SV, Alpers CE, Appel GB, Balow JE, Bruijn JA, Cook T, Ferrario F, et al; International Society of Nephrology Working Group on the Classification of Lupus Nephritis; Renal Pathology Society Working Group on the Classification of Lupus Nephritis: The classification of glomerulonephritis in systemic lupus erythematosus revisited. Kidney Int 65: 521-530, 2004.
18. Li W, Titov AA and Morel L: An update on lupus animal models. Curr Opin Rheumatol 29: 434-441, 2017.

19. Yao G, Qi J, Zhang Z, Huang S, Geng L, Li W, Chen W, Tang X, Wang S and Sun L: Endothelial cell injury is involved in atherosclerosis and lupus symptoms in gld.apoE ${ }^{-/}$mice. Int J Rheum Dis 22: 488-496, 2019.

20. Livak KJ and Schmittgen TD: Analysis of relative gene expression data using real-time quantitative PCR and the 2(- $\Delta \Delta \mathrm{C}(\mathrm{T}))$ Method. Methods 25: 402-408, 2001.

21. Larosa M, Zen M, Gatto M, Jesus D, Zanatta E, Iaccarino L, Inês L and Doria A: IL-12 and IL-23/Th17 axis in systemic lupus erythematosus. Exp Biol Med (Maywood) 244: 42-51, 2019.

22. Zundler S and Neurath MF: Interleukin-12: Functional activities and implications for disease. Cytokine Growth Factor Rev 26: $559-568,2015$

23. Trembleau S, Penna G, Gregori S, Giarratana N and Adorini L: IL-12 administration accelerates autoimmune diabetes in both wild-type and IFN-gamma-deficient nonobese diabetic mice, revealing pathogenic and protective effects of IL-12-induced IFN-gamma. J Immunol 170: 5491-5501, 2003.

24. Smith T, Hewson AK, Kingsley CI, Leonard JP and Cuzner ML: Interleukin-12 induces relapse in experimental allergic encephalomyelitis in the Lewis rat. Am J Pathol 150: 1909-1917, 1997.

25. Constantinescu CS, Wysocka M, Hilliard B, Ventura ES, Lavi E, Trinchieri G and Rostami A: Antibodies against IL-12 prevent superantigen-induced and spontaneous relapses of experimental autoimmune encephalomyelitis. J Immunol 161: 5097-5104, 1998.

26. McIntyre KW, Shuster DJ, Gillooly KM, Warrier RR, Connaughton SE, Hall LB, Arp LH, Gately MK and Magram J: Reduced incidence and severity of collagen-induced arthritis in interleukin-12-deficient mice. Eur J Immunol 26: 2933-2938, 1996.

27. Malfait AM, Butler DM, Presky DH, Maini RN, Brennan FM and Feldmann M: Blockade of IL-12 during the induction of collagen-induced arthritis (CIA) markedly attenuates the severity of the arthritis. Clin Exp Immunol 111: 377-383, 1998.

28. Talaat RM, Mohamed SF, Bassyouni IH and Raouf AA: Th1/Th2/ Th17/Treg cytokine imbalance in systemic lupus erythematosus (SLE) patients: Correlation with disease activity. Cytokine 72 : 146-153, 2015

29. Lauwerys BR, Van Snick J and Houssiau FA: Serum IL-12 in systemic lupus erythematosus: Absence of p70 heterodimers but presence of $\mathrm{p} 40$ monomers correlating with disease activity. Lupus 11: 384-387, 2002.

30. Segal R, Dayan M, Zinger H, Habut B, Shearer GM and Mozes E: The effect of IL-12 on clinical and laboratory aspects of experimental SLE in young and aging mice. Exp Gerontol 38: 661-668, 2003.

31. Neumann D, Tschernig T, Popa D, Schmiedl A, Pérez de Lema G, Resch K and Martin MU: Injection of IL-12- and IL-18encoding plasmids ameliorates the autoimmune pathology of MRL/Mp-Tnfrsf6lpr mice: Synergistic effect on autoimmune symptoms. Int Immunol 18: 1779-1787, 2006.

32. Kikawada E, Lenda DM and Kelley VR: IL-12 deficiency in MRL-Fas(lpr) mice delays nephritis and intrarenal IFN- $\gamma$ expression, and diminishes systemic pathology. J Immunol 170: 3915-3925, 2003

This work is licensed under a Creative Commons Attribution-NonCommercial-NoDerivatives 4.0 International (CC BY-NC-ND 4.0) License. 